\title{
Estudo retrospectivo das complicações associadas à exodontia de terceiros molares em um serviço de referência no sertão paraibano, Brasil
}

Retrospective study of the complications associated with the extraction of third molars

in a referral service in Paraíba, Brazil

Estudio retrospectivo de las complicaciones asociadas a la exodoncia de terceros molares en un servicio de referencia en el interior da Paraíba, Brasil

Manoel Elio Almeida ALVES-FILHO Jaqueline Oliveira BARRETO ${ }^{1}$

Silvestre Estrela da SILVA-JÚNIOR ${ }^{1}$

Julliana Cariry Palhano FREIRE ${ }^{2}$

Julierme Ferreira ROCHA

Eduardo DIAS-RIBEIRO

${ }^{I}$ Departamento de Odontologia do Centro de Saúde e Tecnologia Rural, Universidade Federal de Campina Grande, UFCG, 58708-110 Patos, Paraíba, Brasil ${ }^{2}$ Departamento de Odontologia de Campina Grande, Universidade Estadual da Paraíba, UEPB, 58429-500 Campina Grande, Paraíba, Brasil

${ }^{3}$ Departamento de Cirurgia e Traumatologia Bucomaxilofacial, Universidade Federal de Campina Grande, UFCG, 58708-110 Patos, Paraíba, Brasil

\section{Resumo}

A cirurgia de terceiros molares, embora seja um procedimento rotineiro em nível ambulatorial pode evoluir para complicações trans ou pósoperatorias, as quais o cirurgião deverá estar apto para conduzir os casos da maneira mais adequada. O objetivo desta pesquisa foi avaliar a prevalência das complicações associados a terceiros molares em um serviço de referência no sertão paraibano, Brasil. Este estudo se trata de pesquisa retrospectiva com levantamentos de dados através de prontuários odontológicos, os quais para serem inclusos para análise deveriam estar preenchidos completamente e os pacientes terem se submetidos a exodontias de algum terceiro molar e acompanhado por pelo menos 07 dias pós-operatório. A amostra foi formada com 226 prontuários, os quais registram 483 desses tipos de exodontias. Verificou-se que as complicações tiveram uma prevalência geral de $8,9 \%$, de forma que as mais frequentes foram fratura radicular $(27,9 \%)$, alveolite $(20,93 \%)$, parestesia do nervo alveolar inferior $(18,6 \%)$, parestesia do nervo lingual $(7,0 \%)$, hemorragia trans-operatória $(7,0 \%)$, fratura do túber da maxila (4,65\%), parestesia do nervo facial $(2,32 \%)$, luxação da ATM $(2,32 \%)$, fratura de broca $(2,32 \%)$, hemorragia pós-operatória (2,32\%), laceração de tecido mole (2,32\%), e lipotínea (2,32\%). Conclui-se que os pacientes na faixa etária de 16 a 25 anos, do gênero feminino foram os mais acometidos e a fratura radicular apresentou-se mais prevalente seguida da alveolite e parestesia do nervo alveolar inferior.

Descritores: Prevalência; Cirurgia Bucal; Dente Serotino.

\section{Abstract}

Third molar surgery, although it is a routine outpatient procedure, can progress to trans or postoperative complications, which the surgeon should be able to handle the cases in the most appropriate manner. The aim of this research was to evaluate the prevalence of complications associated with third molars in a referral service Paraiba, Brazil. This study is a retrospective research with data surveys through dental records, which should be fully filled in for analysis and patients underwent exodontia from a third molar and followed up for at least 7 postoperative days. The sample consisted of 226 records, which recorded 483 of these types of exodontia. It was verified that the complications had a general prevalence of $8.9 \%$, so that the most frequent ones were root fracture (27.9\%), alveolitis (20.93\%), paresthesia of the inferior alveolar nerve $(18.6 \%)$, paresthesia of the lingual nerve $(7.0 \%)$, trans-operative haemorrhage $(7.0 \%)$, maxillary tuft fracture (4.65\%), facial nerve paresthesia (2.32\%), TMJ dislocation $(2.32 \%)$, drill fracture $(2.32 \%)$, postoperative bleeding $(2.32 \%)$, laceration of soft tissue $(2.32 \%)$, and lipotine $(2.32 \%)$. It was concluded that female patients aged 16 to 25 years were the most affected and the root fracture was more prevalent followed by alveolitis and inferior alveolar nerve paresthesia.

Descriptors: Epidemiology; Surgery, Oral; Molar, Third.

\section{Resumen}

La cirugía de terceros molares, aunque es un procedimiento rutinario a nivel ambulatorio puede evolucionar para complicaciones trans o post-operatorias, las cuales el cirujano deberá ser apto para conducir los casos de la manera más adecuada. El objetivo de esta investigación fue evaluar la prevalencia de complicaciones asociadas con los terceros molares en un servicio de referencia en el interior da Paraíba, Brasil. Este estudio se trata de una investigación retrospectiva con levantamientos de datos a través de prontuarios odontológicos, los cuales para ser incluidos para análisis deberían ser llenados completamente y los pacientes se sometieron a exodontias de algún tercer molar y acompañado por al menos 07 días post- operativamente. La muestra fue formada con 226 prontuarios, los cuales registran 483 de esos tipos de exodontias. Se observó que las complicaciones tuvieron una prevalencia general del $8,9 \%$, de forma que las más frecuentes fueron fractura radicular (27,9\%), alveolitis (20,93\%), parestesia del nervio alveolar inferior $(18,6 \%)$, parestesia del nervio lingual $(7,0 \%)$, hemorragia transoperatoria $(7,0 \%)$, fractura del túbulo de la mandíbula $(4,65 \%)$, parestesia del nervio facial $(2,32 \%)$, luxación de la ATM $(2,32 \%)$, fractura de broca $(2,32 \%)$, hemorragia postoperatoria $(2,32 \%)$, laceración de tejido blando (2,32\%), y lipotínea (2,32\%). Se concluyó que las pacientes de 16 a 25 años fueron las más afectadas y la fractura de raíz fue más frecuente, seguida de alveolitis y parestesia del nervio alveolar inferior. Descriptores: Epidemiología; Cirugía Bucal; Tercer Molar.

\section{INTRODUÇÃO}

A cirurgia de terceiros molares nos consultórios odontológicos especializados em cirurgia bucomaxilofacial é um dos procedimentos mais comumente realizados ${ }^{1-2}$.

Isso porque esses elementos dentários por serem os últimos a erupcionarem na arcada, apresentam-se como os de maior incidência de inclusão, semi-inclusão ou impactação. Embora seja um procedimento comum, existem complicações trans e pós-operatória que desafiam o cirurgião a conduzir o processo cirúrgico com segurança e confiança para si e seus pacientes ${ }^{3-6}$.

A causa desses dentes não erupcionarem da forma correta na cavidade oral está relacionada com a topografia óssea, espaço da arcada e posição do segundo molar, levando-os a serem indicados para extração. Isso pode gerar acidentes tais como dor, trismo, hemorragias, alveolites, injúrias nervosas, periodontais e a dentes adjacentes, além de infecções em espaços fasciais, fratura da tuberosidade maxilar e/ou mandibular, comunicações bucossinusais entre outros $^{7-8}$. 
Essas complicações podem estar relacionadas ao procedimento em si e por isso o profissional deve estar atento ao preparo do paciente, assepsia, manejo cuidadoso dos tecidos, controle da força aplicada com o instrumental, controle da hemostasia e as adequadas instruções pós-operatórias. Necessita-se também que o cirurgião analise os riscos e os benefícios antes de qualquer tomada de atitude, pois hábitos do paciente, idade, gênero, medicações que fazem uso também podem ser fatores de risco ${ }^{9-10}$.

Considerando que os profissionais que se habilitam em fazer esse tipo de procedimento devem também estar aptos a tratar as possíveis complicações cirúrgicas a que estão sujeitos, e a fim de proporcionar uma melhor preparação na condução dos casos clínicos, o que permite melhor qualidade de atendimento, esse estudo objetivou avaliar a prevalência das complicações associados a terceiros molares em um serviço de referência no sertão paraibano, Brasil.

\section{MATERIAL E MÉTODO}

Este estudo foi aprovado pelo Comitê de Ética em Pesquisa das Faculdades Integradas Patos (CEP-FIP), sob número de protocolo: 2.713.948. Trata-se de uma pesquisa retrospectiva, transversal, descritiva, quantitativa, que envolve o uso de técnicas padronizadas de avaliação de dados (prontuários), sendo por isso dispensado o Termo de Consentimento Livre e Esclarecido (TCLE). Todavia, todos os envolvidos da pesquisa foram esclarecidos quanto a possibilidades de uso dos seus dados para pesquisa e publicação no ato anamnese, concordando e autorizando com TCLE da própria clínica escola de acordo com a Resolução 466/2012.

A amostra foi constituída de 226 prontuários de pacientes submetidos à extração dos terceiros molares na Clínica Escola de Odontologia da Universidade Federal de Campina Grande, Patos, Paraíba, Brasil, coletados no período de 2015 a 2017. Para inclusão das fichas no estudo foi estabelecido extração de, pelo menos, um terceiro molar podendo ser tanto da arcada superior como da inferior; descrição detalhada do procedimento cirúrgico no prontuário; e acompanhamento pós-operatório mínimo de sete dias. Foram excluídos todos aqueles prontuários incompletos de informações necessárias para pesquisa.

Após o levantamento de coleta de dados, 483 extrações foram incluídas no estudo, sendo avaliados o gênero, a idade, os fatores de risco (sistêmicos/hábito) e as complicações associadas as exodontias. Os dados obtidos foram compilados e analisados por meio da estatística descritiva, utilizando o programa Microsoft Office Excel 2013 (Windows $7^{\circledR}$ ). A partir dos dados, foi elaborada a distribuição de frequências de todas as variáveis abordadas no estudo.

\section{RESULTADOS}

Do total de 226 prontuários de pacientes incluídos no estudo, a maioria foi do sexo feminino $(71,68 \%)$ e tinham idades que variaram de 16 a 42 anos. Dessas fichas clínica, foram extraídos 483 terceiros molares, sendo $69,56 \%$ no gênero feminino e $30,43 \%$ no gênero masculino. Com relação às arcadas dentárias foi observada pequena diferença percentual entre os dentes superiores $(50,72 \%)$ com os dentes inferiores $(24,84 \%)$.

A Tabela 1 mostra a relação entre faixa etária e sexo dos pacientes avaliados, de forma didática, os mesmos foram aglutinados em faixas etárias a cada 10 (dez) anos, ficando distribuídas de tal forma: de 16 até 25 anos, de 26 até 35 anos, de 36 até 45 anos. Percebe-se nesse estudo que a maioria dos indivíduos estava na faixa etária de 16 a 25 anos de idade.

Tabela 1: Distribuição de frequência absoluta por gênero e faixa etária.

\begin{tabular}{cccc}
\hline \multicolumn{4}{c}{ Número absoluto de pacientes } \\
\hline Faixa etária & Feminino & Masculino & Total \\
$16-25$ & 101 & 50 & 151 \\
$26-35$ & 52 & 11 & 63 \\
$36-45$ & 09 & 03 & 12 \\
Total & 162 & 64 & 226 \\
\hline
\end{tabular}

Do total de exodontias de terceiros molares analisados, houve uma prevalência de 8,9\% (43 dentes) casos de complicações cirúrgicas, acometendo $63,63 \%$ dos casos a faixa etária de 16 até 25 anos e $36,37 \%$ dos casos na faixa etária de 26 até 35 anos. A Tabela 2 apresenta as principais complicações que foram fratura radicular, alveolite, parestesia do nervo alveolar inferior (NAI) e do nervo lingual, e hemorragias trans-operatórias.

Tabela 2: Número absoluto e percentual de cada complicação.

\begin{tabular}{|c|c|c|}
\hline Complicação & $\begin{array}{l}\text { Número } \\
\text { absoluto (n) }\end{array}$ & $\begin{array}{l}\text { Percentual } \\
\text { (\%) }\end{array}$ \\
\hline Fratura radicular & 12 & 27,9 \\
\hline Alveolite & 9 & 20,93 \\
\hline Parestesia do nervo alveolar inferior & 8 & 18,6 \\
\hline $\begin{array}{l}\text { Parestesia do nervo Lingual; } \\
\text { Hemorragias trans-operatórias }\end{array}$ & 3 & 7,0 \\
\hline Fratura de túber da maxila & 2 & 4,65 \\
\hline $\begin{array}{l}\text { Paralisia do nervo facial; Fratura de } \\
\text { broca; Luxação da ATM; Hemorragias } \\
\text { pós-operatórias; Laceração de tecido } \\
\text { mole; Lipotimia. }\end{array}$ & 1 & 2,32 \\
\hline
\end{tabular}

\section{DISCUSSÃO}

Embora as cirurgias de remoção de terceiros molares sejam preferencialmente do âmbito de atuação do cirurgião bucomaxilofacial, em muitas situações são realizadas por cirurgiões-dentistas generalistas. A despeito do profissional responsável pelo procedimento, destaca-se a importância da cautela pelo risco de complicações trans e pósoperatórias. Logo, devem ser analisados os riscos e os benefícios, além dos grupos de pessoas que necessitam de atenção imediata com seu estado de saúde (grupos de risco) para esses tipos de extrações ${ }^{1,2,4,11}$. 
Ao estudar a literatura cientifica, percebe-se que a prevalência dessas complicações declinou com o tempo, podendo ser justificado pela maior capacidade dos cirurgiões em detectar os casos de maior complexidade e previamente se prepararem para prevenir tais acidentes ${ }^{3,6-8,12}$. Em um estudo retrospectivo, Bui et al. $^{13}$ encontraram taxa de complicações operatórias de 2,2\%, Chiapasco ${ }^{14}$, encontraram uma incidência de $1,1 \%$ de acidentes operatórios e no estudo prospectivo de coorte Chuang $^{2}$, a taxa de complicações operatória foi de $3,9 \%$. Neste estudo com prevalência de $8,9, \log$ o foi maior que estes estudos, todavia, menor que Kato et al. ${ }^{15}$ e Bachmann et al. ${ }^{16}$, que obtiveram respectivamente $10,47 \%, 11 \%$ e $19 \%$ de complicações do total de cirurgias inclusas em seus estudos.

A maioria dos pacientes desta pesquisa eram do sexo feminino, concordando com os estudos de Bachmann et $\mathrm{al}^{16}$, Blondeau e Daniel ${ }^{17}$, Pitekova et al. ${ }^{18}$ e Benediktsdottir et al. ${ }^{19}$, apontando o gênero feminino como o mais acometido por tais problemas.

A faixa etária que possui maior volume de pacientes é a de 16-25 anos, concordando com Kato et. $\mathrm{al}^{15}$. Também esta faixa etária apresentou o maior número de extrações, respondendo por $72,25 \%$ dos procedimentos, indo de encontro com as recomendações de $\operatorname{Hupp}^{20}$ e de outros autores ${ }^{21,22}$ que já defendiam que pacientes mais velhos são mais susceptíveis a sofrerem com complicações e acidentes trans e pós-operatórios, pois estes são acometidos de um atraso de cicatrização e formação de osso em quantidade menor. Em estudo conduzido por Benediktsdóttir et al. ${ }^{19}$, os grupos mais velhos tiveram mais que o dobro do risco de um tempo de operação prolongado do que o grupo mais jovem, o que os coloca no grupo de risco para complicações associadas a estes tipos de exodontia.

A fratura radicular foi a complicação que mais ocorreu, corroborando com outros estudos ${ }^{15,23}$. Enquanto a luxação da ATM nesta pesquisa só foi vista uma vez e a literatura cientifica relata ser um caso raro que pode estar relacionado a anatomia, em ocasiões de fossa mandibular rasa ${ }^{11}$.

Os casos de alveolite foram as complicações encontradas com a segunda maior incidência nesse estudo, assemelhando-se com os achados de outros estudos ${ }^{15,24}$. Há uma possível relação das alveolites com a idade, podendo haver deficiência no processo de reparo e cicatrização nos pacientes mais velhos e uma piora da qualidade do tecido ósseo. Todavia, isso não condiz com os resultados desta pesquisa, pois os casos observados manifestaram em pacientes na faixa etária de 16 a 25 anos, podendo está relacionado com a observação do estudo de AlKhateeb et al. ${ }^{25}$, o qual afirmou que a incidência de alveolite era muito maior $(21,9 \%)$, quando os dentes eram removidos por razões terapêuticas do que por razões profiláticas $(7,1 \%)$, além também da complexidade e duração da cirurgia.

Com relação aos casos de parestesia do nervo alveolar inferior, Kato et al. ${ }^{15}$, Bachmann et $\mathrm{al}^{16}$, Alling $^{26}$ e Mercier e Precious ${ }^{27}$ relatam que estudos prévios apresentam índices de parestesia variando entre $0,4 \%$ e $8,4 \%$, não especificando o tempo da parestesia, observam também que o grau de impacção tem influência direta nos quadros dessa complicação, além das posições mesioangulares e distoangulares apresentarem duas vezes mais chances de provocarem algum grau de parestesia. Quanto a parestesia do nervo lingual pode ser causada por manipulação excessiva do retalho e o uso inadequada dos instrumentos, podendo esta variar de 0 e $23 \%$ de ocorrência $^{15}$, porém nesta pesquisa nota-se que esse dado tem uma prevalência bem menor quando se compara com demais estudos.

Nesta pesquisa foi encontrado valor de 1,65\% de parestesia do nervo alveolar inferior, corroborando com outros achados ${ }^{10,28-30}$. Todos os casos manifestaram no sexo feminino o que se confirma com o estudo de Silveira ${ }^{30}$, este por sua vez explica que este sexo é mais susceptível a proximidade entre os terceiros molares inferiores com o canal mandibular, devido a menor espessura óssea mandibular no sentido vestíbulo-lingual, acarretando uma maior proximidade entre os ápices radiculares dos terceiros molares com o canal mandibular. Entretanto, outros autores não encontraram essa complicação ${ }^{31-33}$.

Em relação à hemorragia, ela pode ocorrer tanto no período transoperatório quanto após o termino da cirurgia, por isso a importância em acompanhar o paciente no seu pós-operatório e orienta-lo de maneira adequada. Os quadros de hemorragia do presente estudo $0,62 \%$ ocorreram no transoperatório e $0,2 \%$ no pós-operatório. No estudo de Sebastiani et $\mathrm{al}^{31}$, também encontraram prevalência baixa para este tipo de complicação e segundo Boulox et al. $^{34}$, a hemorragia é mais prevalente em pacientes mais idosos com dentes profundamente impactados e quatro vezes mais associada a terceiros molares inferiores que os terceiros molares superiores.

Santos et al. ${ }^{35}$ afirmam que a paralisia facial periférica decorrente de cirurgias bucais é considerada incomum e sua causa é desconhecida. Neste estudo houve um caso de paralisia do nervo facial de forma transitória durante o procedimento, muito embora suas funções foram reestabelecidas de maneira natural, não deixando nenhuma sequela ao paciente.

Nas situações em que foi observada a fratura da tuberosidade maxilar, o fragmento ósseo foi removido por apresentar-se aderido ao elemento dentário, sem, contudo, ocasionar problemas aos pacientes, assim como no caso da fratura da broca 
que foi removida no mesmo momento cirúrgico. Em ambos os casos não constatou uso inadequado de força, ou emprego de técnica errônea.

Para Santiago et al. $^{36}$ a lipotimia é a emergência mais relatada pelos cirurgiões dentistas nos consultórios odontológicos, sendo citada em 15\% dos entrevistados. Neste presente levantamento também houve um caso de lipotimia, porém em percentual menor.

De acordo com $\mathrm{Hupp}^{20}$, as complicações associadas a exodontias não estão relacionadas apenas a técnica cirúrgica e habilidade do profissional, mas também aos comprometimentos sistêmicos do paciente e seu comprometimento em seguir as recomendações pós-operatórias, logo, é de fundamental orientar esses indivíduos quantos aos riscos que estão sujeitos.

\section{CONCLUSÃO}

Os pacientes na faixa etária de 16 a 25 anos, do gênero feminino foram os mais acometidos e a fratura radicular apresentou-se mais prevalente seguida da alveolite e parestesia do nervo alveolar inferior.

\section{REFERÊNCIAS}

1. Costa MG, Pazzini CA, Pantuzo MCG, Jorge MLR, Marques LS. Is there justification for prophylactic extraction of third molars? A systematic review. Braz Oral Res. 2013; 27(2):183-88.

2. Chang SK, Perrott DH, Susarla SM, Dodson TB. Age as a risk factor for third molar surgery complications. J Oral Maxillofacial Surg. 2007; 65(9):1685-92.

3. Haug RH, Perrott DH, Gonzalez ML, Talwar RM. The American Association of Oral and Maxillofacial Surgeons Age-Related Third Molar Study. J Oral Maxillofac Surg. 2005; 63:1106-14.

4. Mahdey HM, Arora S, Wei M. Prevalence and difficulty index associated with the mandibular molar impaction among malaysian ethnicities: a clinico-radiographic study. J Clin Diagn Res. 2015;9(9):ZC65-8.

5. Dias-Ribeiro E, Lima-Júnior JL, Barbosa JL, Haagsma IB, Lucena LBS, Marzola C. Avaliação das posições de terceiros molares retidos em relação à classificação de Winter. Rev Odontol UNESP. 2008;37(3):203-9.

6. Kanneppady SK, Balamanikandasrinivasan, Kumaresan R, Sakri SB. A comparative study on radiographic analysis of impacted third molars among three ethnic groups of patients attending AIMST Dental Institute, Malaysia. Dent Res (Isfahan). 2013;10(3):353-58.

7. Reddy KVG, Prasad KVV. Prevalence of third molar impactions in urban population of age 22-
30 years in South India: an epidemological study. J Indian Dent Assoc. 2011;5(5):609-11.

8. Al-Anqudi SM, Al-Sudairy S, Al-Hosni A, AlManiri A. Prevalence and pattern of third molar impaction - a retrospective study of radiographs in Oman. Sultan Qaboos Univ Med J. 2014;14(3):e388-e92.

9. Susarla SM, Dodson TB. Risk factors for third molar extraction difficulty. J Oral Maxillofac Surg. 2004;62(11):1363-71.

10.Sursala MS, Blaeser BF, Magalnick D. Third molar surgery and associated complication. Oral Maxillofacial Surg Clin North Am. 2003; 15(2):177-86.

11.de Carvalho RW, de Araújo Filho RC, do Egito Vasconcelos BC. Assessment of factors associated with surgical difficulty during removal of impacted maxillary third molars. J Oral Maxillofac Surg. 2013;71(5):839-45.

12. Osborn TP, Frederickson G Jr, Small IA, Torgerson TS. A Prospective study of complications related to mandibular third molar surgery. J Oral Maxillofac Surg. 1985; 43(10):767-69.

13.Bui, CH, Seldin EB, Dodson TB. Types, frequencies, and risk factors for complications after third molar extraction. J. Oral Maxillofac Surg. 2003;61(12):1379-89.

14. Chiapasco M, De Cicco L, Marrone G. Side effects and complications associates with third molar surgery. Oral Surg Oral Med Oral Pathol. 2006; 76(4):412-20.

15. Kato RB, Bueno RBL, Oliveira Neto PJ, Ribeiro MC, Azenha MR. Acidentes e complicações associadas á cirurgia dos terceiros molares realizada por alunos de odontologia. Rev cir traumatol buco-maxilo-fac. 2010;10(4):45-54.

16.Bachmann H, Cáceres R, Muñoz C, Uribe S. Complicaciones en cirugía de terceros molares entre los años 2007-2010, en un hospital urbano, Chile. Int J Odontostomat. 2014;8(1):107-12.

17. Blondeau F, Daniel NG. Extraction of impacted mandibular third molars: postoperative complications and their risk factors. J Can Dent Assoc. 2007;73(4):325.

18.Pitekova, L.; Satko, I. \& Novotnakova, D. Complications after third molar surgery. Bratisl Lek Listy. 2010;111(5):296-98.

19.Benediktsdóttir IS, Wenzel A, Petersen JK, Hintze H. Mandibular third molar removal: Risk indicators for extended operation time, postoperative pain, and complications. Oral Surg, Oral Med, Oral Pathol, Oral Radiol, Endod. 2004; 97(4):438-46.

20.Hupp JR. Cirurgia oral maxilofacial contemporânea. 6 . ed. Rio de Janeiro: Elsevier; 2015. 
21.Laskin D. Oral and Maxillofacial Surgery. St Louis:Mosby Co; 1985.

22. Killey HC, Kay LW. The impacted wisdom tooth. Edinburgh: Churchill Livingstone; 1975.

23. Araujo OC, Agostinho CNLF, Marinho LMRF, Rabêlo LRS, Bastos EG, Silva VC. Incidência dos acidentes e complicações em cirurgias de terceiros molares. Rev Odontol UNESP. 2011;40(6):290-95.

24. Oliveira LB, Schmidt DB, Assis AF, Gabrielli MAC, Hochuli-Vieira EH, Pereira Filho VAP. Avaliação dos acidentes e complicações associados à exodontias dos $3^{\circ}$ molares. Rev cir traumatol buco-maxilo-fac. 2006;6(2):51-6.

25.al-Khateeb TL, El-Marsafi AI, Butler NP. The relationship between the indications of the surgical removal of impacted third molars and incidence of alveolar osteitis. J Oral Maxillofac Surg. 1991;49(2):141-45.

26. Alling CC 3rd. Dysesthesia of the lingual and inferior alveolar nerves following third molar surgery. J Oral Maxillofac Surg. 1986;44(6):454-57.

27. Mercier P, Precious D. Risk and benefits of removal of impacted third molars: a critical review of the literature. J Oral Maxillofac. Surg. 1992;21(1):17-27.

28.Cheung LK, Leung YY, Chow LK, Wong MC, Chan EK, Fok YH. Incidence of neurosensory deficits and recovery after lower third molar surgery: a prospective clinical study of 4,338 cases. Int J Oral Maxillofac Surg. 2010; 39(4):320-26.

29. Contar CM, de Oliveira P, Kanegusuku K, Berticelli RD, Azevedo-Alanis LR, Machado MA. Complications in third molar removal: a retrospective study of 588 patients. Med Oral Patol Oral Cir Bucal. 2010;15(1):e74-8.

30. Silveira KG, Costa FWG, Bezerra MF, Pimenta AVM, Carvalho FSR, Soares ECS. Sinais radiográficos preditivos de proximidade entre terceiro molar e canal mandibular através de tomografia computorizada. Rev Port Estomatol Med Dent Cir Maxilofac. 2016;57(1):30-7.

31. Sebastiani' AM, Todero SRB, Gabardo G, Costa DJ, Rebelatto NLB, Scario R. Intraoperative accidents associated with surgical removal of third molars. Braz J Oral Sci. 2014;13(4):276-80

32.Wofford DT, Miller RI. Prospective study of dysesthesia following odontectomy of impacted mandibular third molars. J Oral Maxillofac Surg. 1987;45(1):15-9.

33.Swanson AE. Removing the mandibular third molar: neurosensory deficits and consequent litigation. J Can Dent Assoc. 1989;55(5):383-86.

34.Bouloux GF, Steed MB, Perciaccante VJ. Complications of third molar surgery. Oral Maxillofacial Surg Clin North Am. 2007; 19(1):117-28.

35.Santos MESM, Martins CAM, Beltrao GC, Gallo
TB. Paralisia do nervo facial após remoção de enxerto mandibular- relato de caso. Rev cir traumatol buco-maxilo-fac. 2006;6(3):33-8.

36. Santiago JA, Martins Neto RS, Lima VN, Queiroz SBF, Carvalho ACGS, Magro Filho O. Avaliação dos cirurgiões-dentistas de Quixerobim sobre emergências médicas em consultório odontológico. Braz J Surg Clin Res - BJSCR. 2016, 13(1):23-8.

\section{CONFLITO DE INTERESSES}

Os autores declaram não haver conflitos de interesse.

\section{AUTOR PARA CORRESPONDENCIA}

\section{Jaqueline Oliveira Barreto}

jacquinha_barreto@hotmail.com

Submetido em 18/11/2018

Aceito em 12/03/2019 